\title{
Safety in anesthesia: quasi-experimental study for the evaluation of the impact of a multimodal strategy on the registry of critical incidents
}

Garrido $A^{1}$, Arnal $D^{2}$, Portas $M^{1}$, Jiménez $A B^{3}$, Chamorro $E^{1}$, Zaballos $M^{1}$, SENSAR (Sistema Español de Notificación en Seguridad en Anestesia y Reanimación) y GTSA (Grupo de trabajo en Seguridad en Anestesia del Hospital General Universitario Gregorio Marañón)

${ }^{1}$ Hospital General Universitario Gregorio Marañón - Madrid (Spain) ${ }^{2}$ Hospital Universitario Fundación Alcorcón - Madrid (Spain), ${ }^{3}$ Hospital Universitario Severo Ochoa - Madrid (Spain)

Background and Goal of Study: A Critical Incident Reporting System (CIRS) improves Patient Safety (PS) by using the systematic analysis of the latent factors (LF), proposing and executing improvement measures (IM) that prevent the repetition of the Cls. Its use is correlated with a modern Culture of Safety (CS). Several initiatives have been launched to increase its use but there are not any conclusive studies so far that enable drawing conclusions to the clinical practice. The Spanish Anesthesia and Recovery Safety Notification System (SENSAR) is a CIRS created in 2009. Five years after, a multimodal strategy (MMS) was implemented to improve its performance. Goal: To evaluate the impact of a MMS developed by SENSAR in the evolution of $\mathrm{Cl}$ reporting in anesthesiology by comparing two periods (2009-2013 and 20142016).

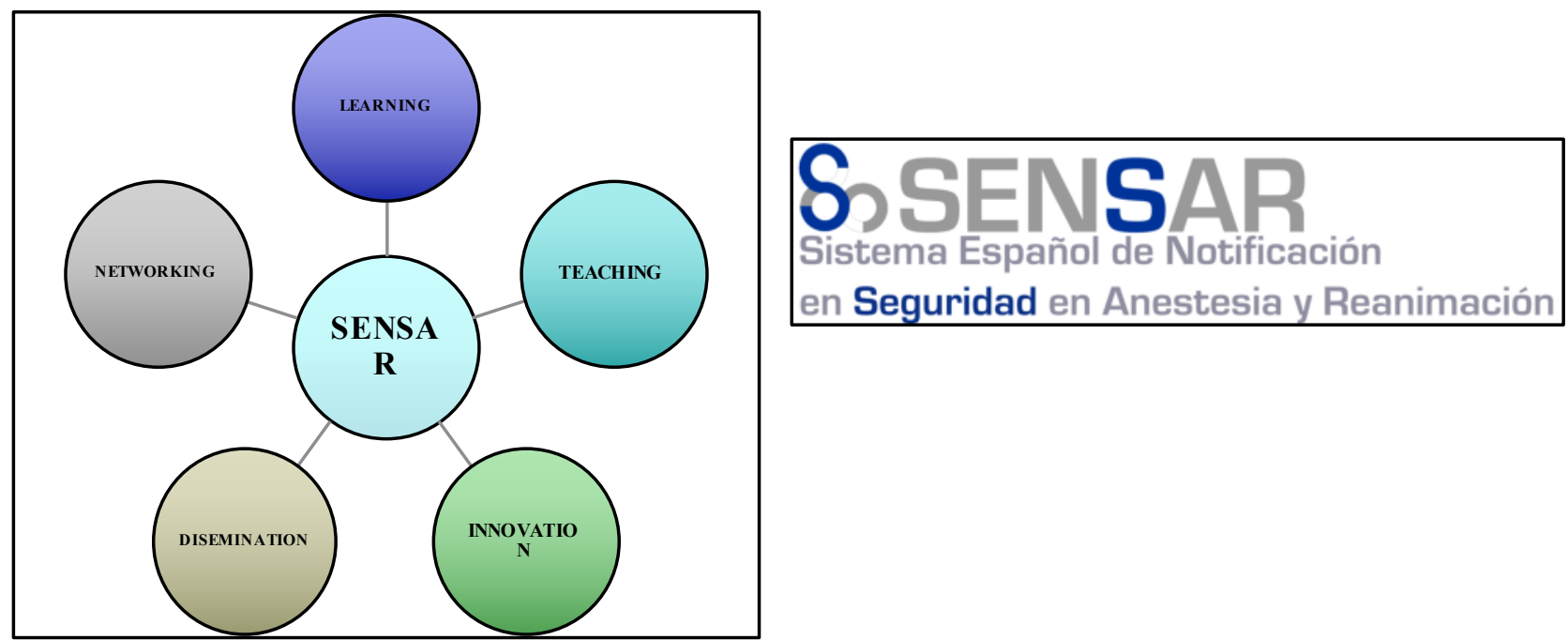

Materials and Methods: Data from the SENSAR database "ANESTIC" of communication and analysis. A quasiexperimental analytic study was designed on the activity data gathered in a prospective manner and analyzed in a retrospective manner. The MMS intervention was defined as the set of actions taken five years after its establishment. Both periods were compared.

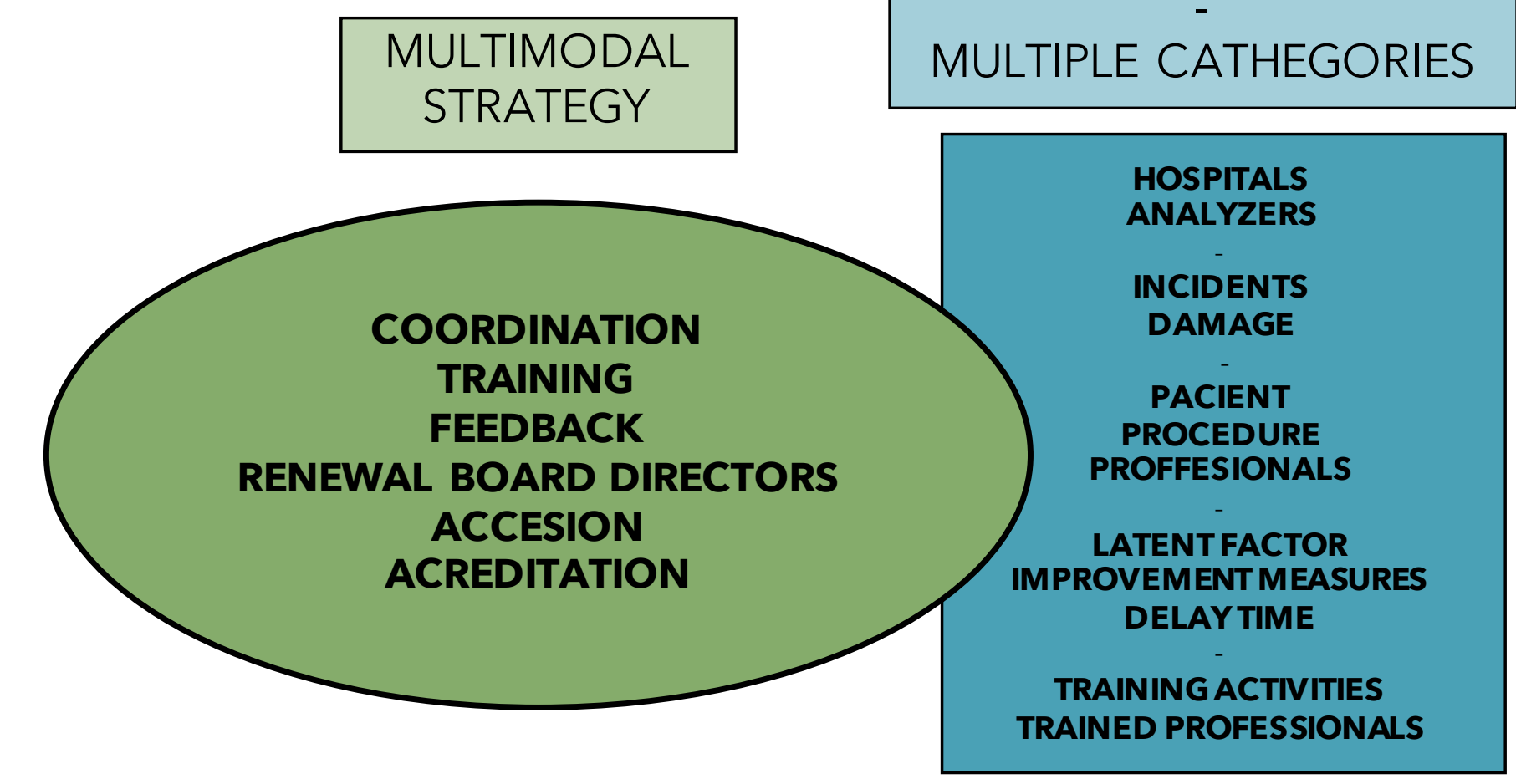

Results and Discussion: At the end of the research period, 92 hospitals had joined, 84 had reported, 75 had conducted analysis. 497 local analyzers were identified. 7,232 reported $\mathrm{CI}$ were registered, $\mathbf{6 , 4 4 6}$ of which (89.1\%) were analyzed. The cases were mainly of $\mathrm{Cls}$ with no damage $(77.3 \%)$. Specialist anesthesiologists were the main reporters (87.3\%) opposite to nurses (4\%). A total of $\mathbf{1 2 , 7 8 2}$ IM proposals were identified. The comparative analysis offered statistically significant differences in favor of the second period with an increase in the number of hospitals that account for $75 \%$ of the reports and the analysis ( $\Delta 20.7 \%$ and $5.4 \%$, respectively), with a decrease in inactivated hospitals $(\Delta-72.45 \%)$, and with an increase of hospitals outside the national territory (4 hospitals). There was an increase in the reported Cls per year ( $\Delta 67.96 \%)$, shared with other hospitals $(\Delta 4.49 \%)$ and presented in session $(\Delta 23.12 \%)$. The delay times of the $\mathrm{Cl}$ analysis and the execution of measures were reduced in 40.43 and 54.48 days, respectively.
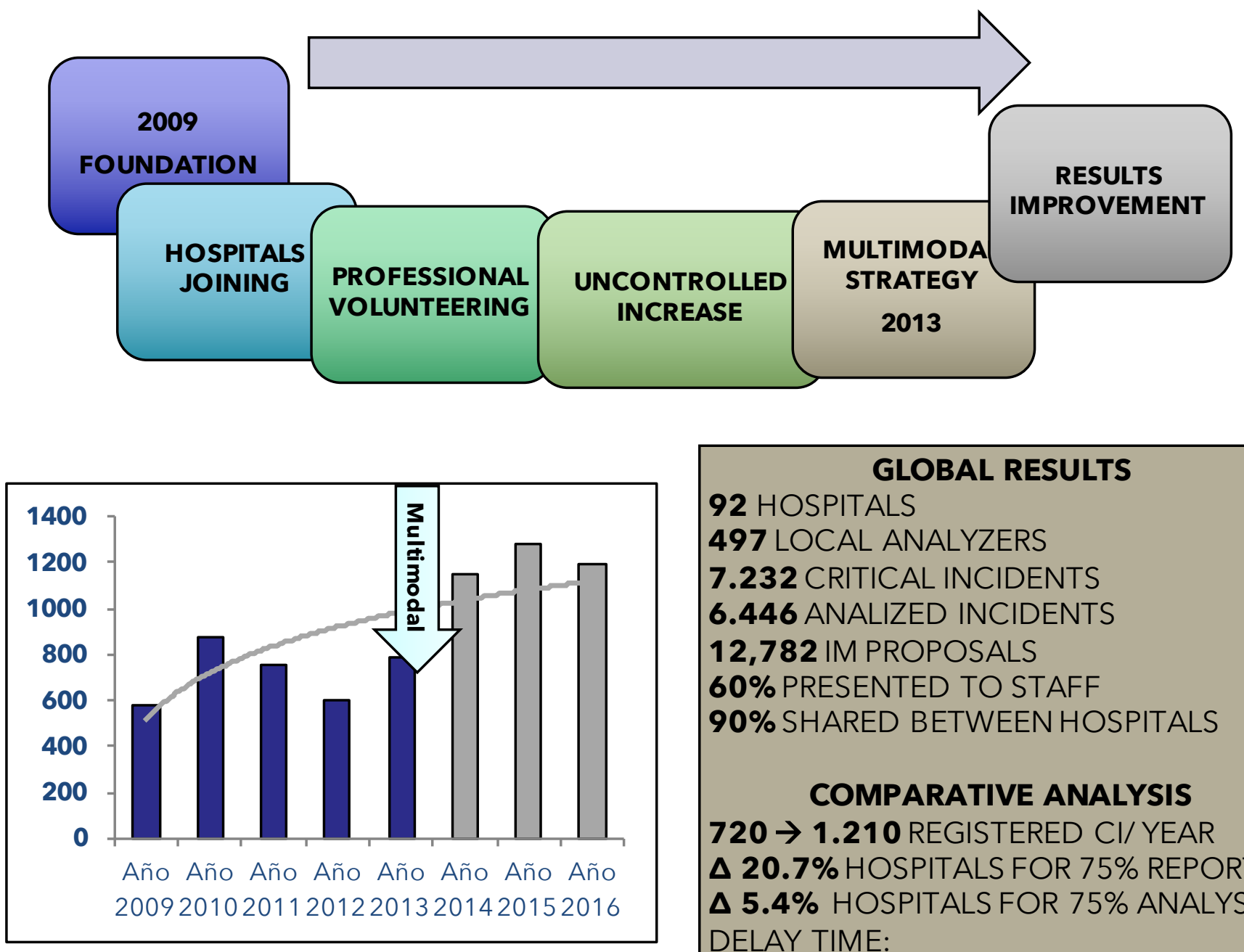

$$
\begin{aligned}
& \mathbf{9 2} \text { HOSPITALS } \\
& \mathbf{4 9 7} \text { LOCAL ANALYZERS } \\
& \mathbf{7 . 2 3 2} \text { CRITICAL INCIDENTS } \\
& \mathbf{6 . 4 4 6} \text { ANALIZED INCIDENTS } \\
& \mathbf{1 2 , 7 8 2} \text { IM PROPOSALS } \\
& \mathbf{6 0 \%} \text { PRESENTED TO STAFF } \\
& \mathbf{9 0 \%} \text { SHARED BETWEEN HOSPITALS } \\
& \\
& \text { COMPARATIVE ANALYSIS } \\
& \mathbf{7 2 0} \rightarrow \mathbf{1 . 2 1 0} \text { REGISTERED CI/ YEAR } \\
& \mathbf{2 0 . 7 \%} \text { HOSPITALS FOR 75\% REPORTS } \\
& \mathbf{5} \mathbf{5 . 4 \%} \text { HOSPITALS FOR 75\% ANALYSIS } \\
& \text { DELAY TIME: } \\
& \mathbf{1 0 7} \rightarrow \mathbf{6 6} \text { DAYS UNTIL ANALYSIS } \\
& \mathbf{8 8} \rightarrow \mathbf{3 3} \text { DAYS UNTIL EXECUTION }
\end{aligned}
$$

Conclusions: The positive impact of the developed MMS by SENSAR has meant an improvement of the PS in the field of anesthesiology and related areas, of the CS of professionals and institutions and an improvement of the general and specific performance of the SENSAR organisation to lead institutions in learning from errors. 